\title{
Serial dependence in vision: Merely encoding the previous-trial target is not enough
}

\author{
Gi-Yeul Bae ${ }^{1}$ (D) Steven J. Luck ${ }^{2}$ \\ Published online: 2 January 2020 \\ (C) The Psychonomic Society, Inc. 2020
}

\begin{abstract}
The reported perception of a visual stimulus on one trial can be biased by the stimulus that was presented on the previous trial. In the present study we asked whether encoding the previous-trial stimulus is sufficient to produce this serial dependence effect, or whether the effect also depends on postencoding processes. To distinguish between these possibilities, we designed a task in which participants reported either the color or the direction of a set of colored moving dots on each trial. The to-be-reported dimension was indicated by a postcue after stimulus offset, so participants were required to encode both features of every stimulus. We assessed serial dependence for motion perception as a function of which feature dimension had been reported on the previous trial. In Experiment 1, we found a serial dependence effect for motion only when participants had reported the direction of motion on the previous trial, and not when they had encoded the direction of motion but reported the color of the stimulus. Experiment 2 confirmed that this pattern of results was not driven by the difficulty of the color task. When we used the same response modality for both motion and color reports in Experiment 3, we found significant serial dependence effects following both color-report and motion-report trials, but the effect was significantly weaker following color-report trials. Together, these findings indicate that postperceptual processes play a critical role in serial dependence and that the mere encoding of the previous-trial target is not sufficient to produce the serial dependence effect.
\end{abstract}

Keywords Motion perception $\cdot$ Priming $\cdot$ Attention $\cdot$ Working memory $\cdot$ Serial dependence

Our current thoughts and behaviors are influenced by our past experiences. In the case of visual perception, studies of serial dependence have shown that perceptual decisions for various types of visual stimuli-including simple visual features (e.g., orientation and spatial location) and more complex objects (e.g., faces) - are systematically biased by recent stimuli, even when those stimuli are no longer task-relevant (Bae \& Luck, 2019; Fischer \& Whitney, 2014; Liberman, Fischer, \& Whitney, 2014; Papadimitriou, Ferdoash, \& Snyder, 2015). Despite the ubiquity of such effects, little is known about the mechanism by which past visual experiences influence the processing of new visual inputs. In particular, although

Gi-Yeul Bae

giyeulbae@asu.edu

1 Department of Psychology, Arizona State University, Tempe, AZ, USA

2 Center for Mind \& Brain and Department of Psychology, University of California, Davis, CA, USA researchers assume that serial dependence is driven by the perception of the previous stimuli, the operation of postperceptual processes may also be essential.

Most previous studies of serial dependence required participants to report a single target feature dimension on every trial, which confounds the perception of this feature with the report of the feature. Some studies, however, have included a condition in which no explicit response was required on some trials, which could potentially unconfound the perception and report of the target feature (Czoschke, Fischer, Beitner, Kaiser, \& Bledowski, 2019; Fischer \& Whitney, 2014; Suárez-Pinilla, Seth, \& Roseboom, 2018). These studies showed that serial dependence was present even when the target feature had not been reported on the previous trial, suggesting that serial dependence can be produced by the mere encoding of the previous-trial stimulus. However, because participants were frequently required to report the target feature, it is possible that participants prepared a response even if none was required on the current trial, and that some aspect of response preparation produced the serial dependence effect. 
A recent study used a stimulus with two feature dimensions and tested whether serial dependence was present for one feature dimension when the other feature dimension had been reported on the previous trial (Suárez-Pinilla et al., 2018). The participants in this study saw a random-dot kinematogram (RDK) and reported either the mean or the variance of the dot directions on a given trial. A core finding was that, even though participants saw both the variance and the direction, serial dependence in visual variance occurred only when participants had reported visual variance on the previous trial. However, it should be noted that the study used a precue paradigm in which the relevant feature dimension on a given trial was known to participants prior to stimulus onset. Consequently, feature-based attention might have attenuated the perceptual or memory encoding of the unattended dimension. That is, the lack of serial dependence might have reflected a reduction in the encoding of the other feature dimension, rather than a lack of the report of this dimension.

In the present study, participants had to perceive and remember both features of a two-dimensional stimulus on every trial, but they were asked to report only one of the two features on a given trial. Specifically, participants saw an RDK with colored dots for a short period of time and were then cued to report either the direction or the color of the dots (Fig. 1a). Crucially, color-report and direction-report trials were randomly intermixed, and the relevant dimension was postcued after stimulus offset. Consequently, participants had to perceive and remember both the direction and the color of the dots on each trial to perform the task accurately. This made it possible to test whether stimulus information that had been encoded ${ }^{1}$ but remained unused on the previous trial would still bias the processing of the next stimulus.

If encoding the stimulus is sufficient to produce serial dependence, then serial dependence for motion direction should be observed even when participants reported color on the previous trial. However, if additional report-related processes are necessary for serial dependence to occur, then serial dependence for motion direction should be reduced or eliminated when color was reported on the previous trial. In a series of three experiments, we found that serial dependence for motion direction was largely absent following color-report trials. These results demonstrate that encoding a feature is not sufficient for serial dependence and that postencoding processes play a key role.

\footnotetext{
${ }^{1}$ We use the term encoding to refer to both the perception and storage of a stimulus in memory, without any commitment to which of these encoding processes produces serial dependence effects.
}

\section{Experiment 1}

\section{Method}

Participants Twenty-four college students between the ages of 18 and 30 participated (13 female, 11 male). The sample size was determined a priori on the basis of published studies of serial dependence (Bae \& Luck, 2019; Fischer \& Whitney, 2014). The study was approved by the UC Davis Institutional Review Board, and the participants gave informed consent.

Stimuli and tasks Stimuli were generated in Matlab (The MathWorks, Inc.) using Psychtoolbox (Brainard, 1997; Pelli, 1997) and were presented at $60 \mathrm{~Hz}$ on an LCD monitor (Dell $\mathrm{U} 2412 \mathrm{M})$ with a white background $\left(87.6 \mathrm{~cd} / \mathrm{m}^{2}\right)$ at a viewing distance of $70 \mathrm{~cm}$. A white fixation dot $\left(87.6 \mathrm{~cd} / \mathrm{m}^{2}\right)$ was continuously visible on a black disk $\left(5^{\circ}\right.$ diameter, $<.1 \mathrm{~cd} /$ $\mathrm{m}^{2}$ ) at the center of the screen, except during the intertrial interval (see Fig. 1a).

We used a standard RDK algorithm to generate the motion stimulus (Roitman \& Shadlen, 2002). The stimuli consisted of three groups of dots (dot diameter $=0.3^{\circ}$ ) that were randomly distributed within the black disk (16.7 dots per square degree per second). We used a larger-than-typical dot size in order to increase the discriminability of the dot colors. Each group of dots was presented for one video frame ( $16.67 \mathrm{~ms} /$ group), and the dots were replotted in new locations after a two-frame delay. Thus, the coherent motion was created by the correspondence between the dots in frame $n$ and in frame $n+3$. When the new location of a given dot was outside the black disk, that dot was replotted at a random location on the circumference of the black disk, to maintain dot density. The coherence level was set to $100 \%$, and the speed of motion was set to $6 \%$.

As is illustrated in Fig. 1a, each trial began with the appearance of the fixation dot for $500 \mathrm{~ms}$ on the black disk. The RDK was then presented for $500 \mathrm{~ms}$ (30 frames). The direction of motion on a given trial was one of 16 discrete values equally spaced around the $360^{\circ}$ of possible directions (from $11.25^{\circ}$ to $348.75^{\circ}$, in steps of $22.5^{\circ}$ ). The color of the dots on a given trial was chosen from a set of 180 colors that formed a circle in the CIE $\mathrm{L}^{*} \mathrm{a}^{*} \mathrm{~b}^{*}$ color space $\left(\mathrm{L}^{*}=70, \mathrm{a}^{*}=0, \mathrm{~b}^{*}=0\right.$, radius $=39$, sampled in steps of $2^{\circ}$ ). Participants were asked to remember both the exact direction of motion and the exact color of the dots on every trial.

After the RDK, participants reported either the direction of motion or the color of the dots. The to-be-reported feature was indicated by a white postcue letter (" $\mathrm{D}$ " for direction or " $\mathrm{C}$ " for color; $87.6 \mathrm{~cd} / \mathrm{m}^{2} ; 0.8^{\circ} \times 1.0^{\circ}$ for width $\times$ height) presented at the center of the black disk for $500 \mathrm{~ms}$. When a direction report was indicated, a mouse cursor appeared at the center of the aperture. As soon as participants moved the mouse cursor, 
a

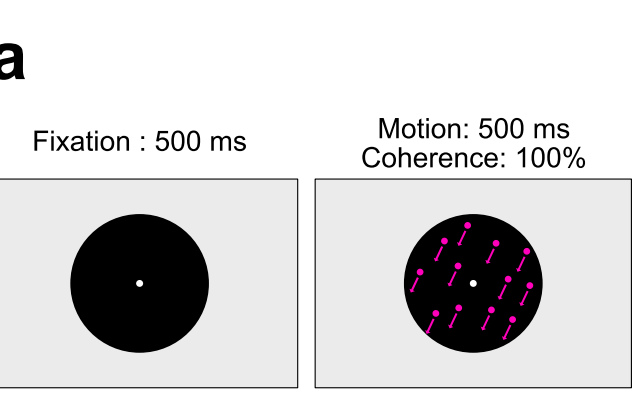

Motion direction: one of 16 directions from $360^{\circ}$ space

Dot color: a random color from a color wheel

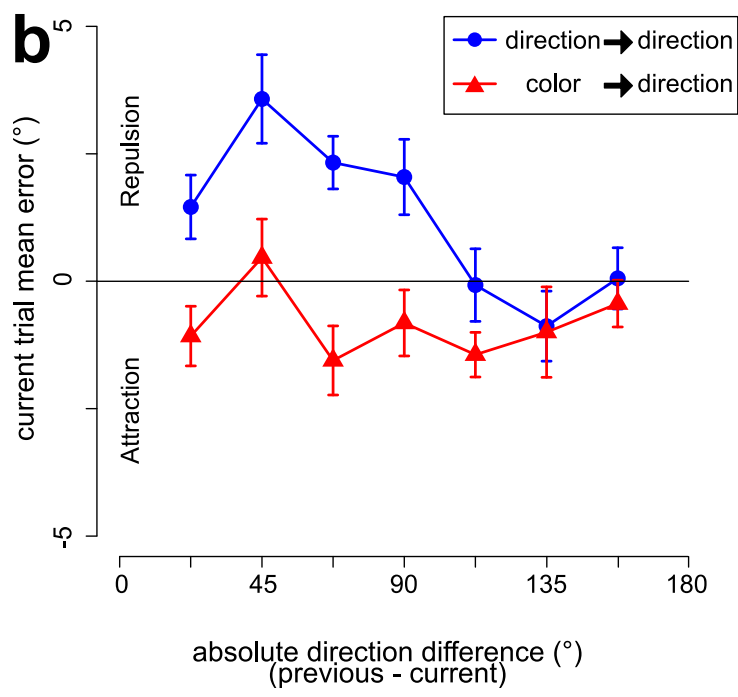

Fig. 1 Experiment 1 task and results. (a) Sequence of stimuli. On each trial, participants saw a random-dot kinematogram (RDK) drawn in colored dots, with a random direction of motion and a random color. The line segments shown in the figure represent the direction of motion and were not visible. After the RDK, a postcue indicated which feature dimension - direction of motion or color - should be reported. When the direction cue (D) was presented, participants continuously adjusted the orientation of a white response line using a mouse to report the direction of motion of the RDK. When the color cue (C) was presented, two test colors were shown, and the participant pressed a key to report the color of the RDK. (b) Mean response errors for the direction report as a function of
Direction Report cue: $500 \mathrm{~ms}$

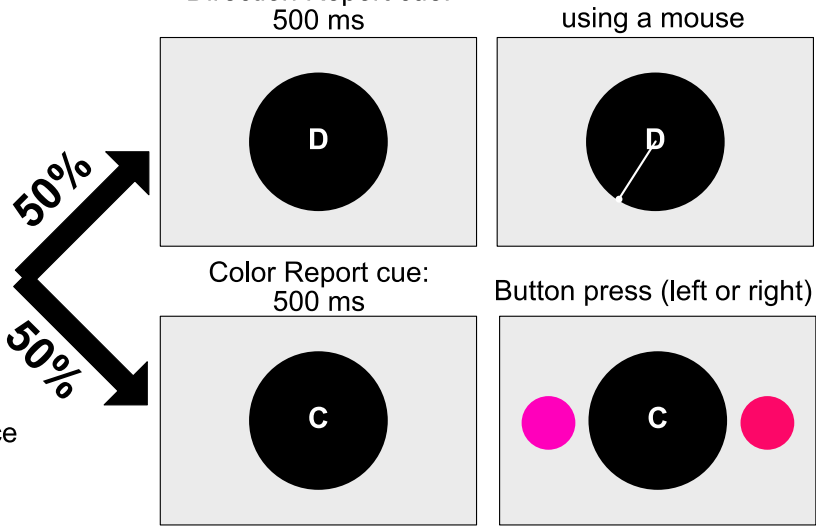

Direction estimation using a mouse

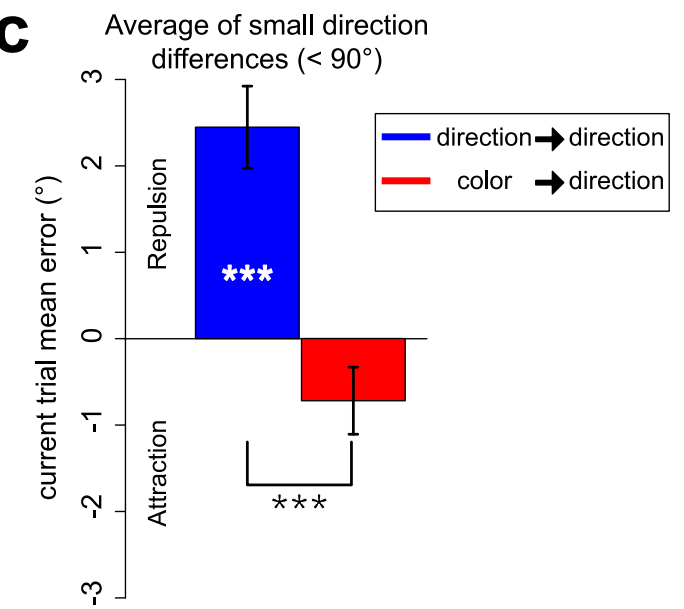

the absolute direction difference between the current and previous trials, plotted separately for trials preceded by a direction report and for trials preceded by a color report. Positive values indicate that the current-trial direction report was biased away from the direction of the previous trial, and negative values indicate that the current-trial direction report was biased toward the direction of the previous trial. (c) Mean response errors for the direction report, averaged across small direction differences $\left(22.5^{\circ}-67.5^{\circ}\right)$, plotted separately for trials preceded by a direction report and trials preceded by a color report. Error bars indicate \pm 1 SEM. ${ }^{* * *} p<.001$. a white line connecting the center of the disk and a point on a circumference of the disk appeared. The orientation of the white line was continuously updated depending on the orientation created by the cursor and the center of the screen, allowing observers to adjust the orientation of the white line to the perceived motion direction. The observer finalized the direction report by clicking a mouse button. When a color report was indicated, two colored circles (diameter $1.5^{\circ}$ ) were presented at $4^{\circ}$ to the left and right of the fixation dot. The color of one of the circles was always identical to the color of the dots in the RDK from that trial. The color of the other circle was 10, 20, or 40 steps away (in either direction, varied randomly across trials) from the true dot color. Observers pressed either the left or the right arrow key on a computer keyboard to report which of the two colors was identical to the color of the dots. The correct color was equally likely to appear on the left and right sides. We used this simple twoalternative forced choice (2AFC) color task to avoid any possible interference between the color task and the motion task. The postcue remained visible until the response was completed. The next trial began after a 1,000-ms intertrial interval, during which only the black disk was visible.

After 16 practice trials, each participant completed 480 experimental trials, with exactly 30 trials for each of the 16 
directions of motion. This included 240 direction-report trials intermixed with 240 color-report trials. The order of the trials was randomly shuffled. Participants took a short break after each block of 48 trials.

Data analysis The main question of the present study was whether the reported direction on a given trial was influenced by the direction of motion on the previous trial. To answer this question, we computed the direction difference between a given trial and its preceding trial. The first trial in each block was necessarily excluded (a total of ten trials per participant). We used 16 discrete motion directions, and there were also 16 discrete direction differences. We used the absolute direction difference in order to increase the number of trials in each cell of the design, resulting in nine different absolute direction differences $\left(0^{\circ}, 22.5^{\circ}, 45.0^{\circ}, 67.5^{\circ}, 90.0^{\circ}, 112.5^{\circ}, 135.0^{\circ}\right.$, $\left.157.5^{\circ}, 180.0^{\circ}\right)$.

Our main dependent variable was the response error, defined as the difference between the reported direction and the true motion direction for the current trial. We coded the sign of this difference so that the response error reflected the bias relative to the direction of motion in the previous trial. Positive values indicated that the direction report was biased away from the previous-trial direction, and negative values indicated that the direction report was biased toward the previous-trial direction. The recoded response errors were then averaged separately for each of the nine direction differences. We excluded trials with a direction difference of $0^{\circ}$ or $180^{\circ}$, because response bias relative to the previous trial is undefined for those trials. We also excluded trials on which the current-trial response error was larger than $60^{\circ}(0.38 \%$ of the direction-report trials), which were likely to reflect lapses of attention.

Following previous research (Bae \& Luck, 2017, 2019), we expected that the reported direction on the current trial would be repelled away from the previous-trial direction, especially when the two directions were similar (e.g., $<90^{\circ}$ ). We therefore assessed the presence of a serial dependence effect by averaging together the direction error values for the smalldirection-difference trials (i.e., $22.5^{\circ}, 45.0^{\circ}$, and $67.5^{\circ}$ ) and comparing this value to zero using a one-sample, two-tailed $t$ test. $^{2}$

\section{Results and discussion}

The mean accuracy for the color task was $87.6 \%($ SEM $=0.7)$, demonstrating that participants successfully encoded color information into working memory on a large proportion of trials.

\footnotetext{
${ }^{2}$ We also performed derivative-of-Gaussian analyses, following previous research (Fischer \& Whitney, 2014), and found the same pattern of results in all three experiments.
}

Figure $1 \mathrm{~b}$ shows the mean response error for the motionreport trials, separately for trials preceded by a direction report and for trials preceded by a color report. When the direction difference between the previous trial and the current trial was smaller than $90^{\circ}$, the reported direction on a given trial was biased away from the direction in the previous trial, but only when the preceding trial was a direction-report trial. There was little or no evidence of serial dependence on motion-report trials when the preceding trial was a color-report trial, even though participants necessarily encoded both direction and color on every trial.

Following previous research (Bae \& Luck, 2017), we quantified the serial dependence effect by averaging the motion-report errors across the trials with direction differences of less than $90^{\circ}$ (i.e., direction differences of $22.5^{\circ}, 45.0^{\circ}$, and $67.5^{\circ}$ ). Figure $1 \mathrm{c}$ shows this averaged value for trials preceded by a direction report and for trials preceded by a color report. When we compared these values to zero with one-sample $t$ tests, we found that trials preceded by a direction report produced a significant repulsion bias $[t(23)=5.150, p=3.219 \mathrm{e}-$ 05 , Cohen's $d=1.486]$, whereas trials preceded by a color report showed a hint of an attraction bias that did not reach significance $[t(23)=-1.855, p=.076$, Cohen's $d=-0.535]$. In addition, a paired $t$ test showed that the serial dependence effect was significantly greater for trials preceded by a direction report than for trials preceded by a color report $[t(23)=$ $6.011, p=3.947 \mathrm{e}-06$, Cohen's $d=1.227]$.

These findings provide clear evidence that, at least in the case of motion direction, encoding a stimulus dimension on one trial is not sufficient to bias the reported value of that dimension on the next trial. Instead, some postencoding process is necessary for the perceived value on one trial to bias performance on the next trial.

\section{Experiment 2}

The color-report task in Experiment 1 was quite difficult, and this may have produced interference with the motion representation on that trial, preventing serial dependence on the next trial. Experiment 2 therefore used an easier color task (Fig. 2a).

\section{Method}

The methods of Experiment 2 were identical to those of Experiment 1, except as noted. A new group of 24 college students participated (21 female, three male).

Instead of being drawn from a set of 180 possible colors, the dots in Experiment 2 were always either green (RGB = $[68,190,170])$ or pink (RGB = [236, 143, 173]; see Fig. 2a). The two options at the time of test on color-report trials were also green and pink. Each participant completed 448 
a

a

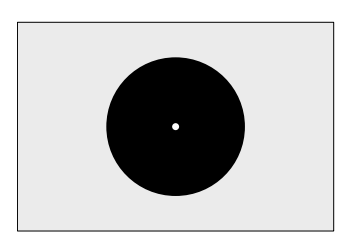

Motion direction: one of 16 directions from $360^{\circ}$ space Dot color: pink or green

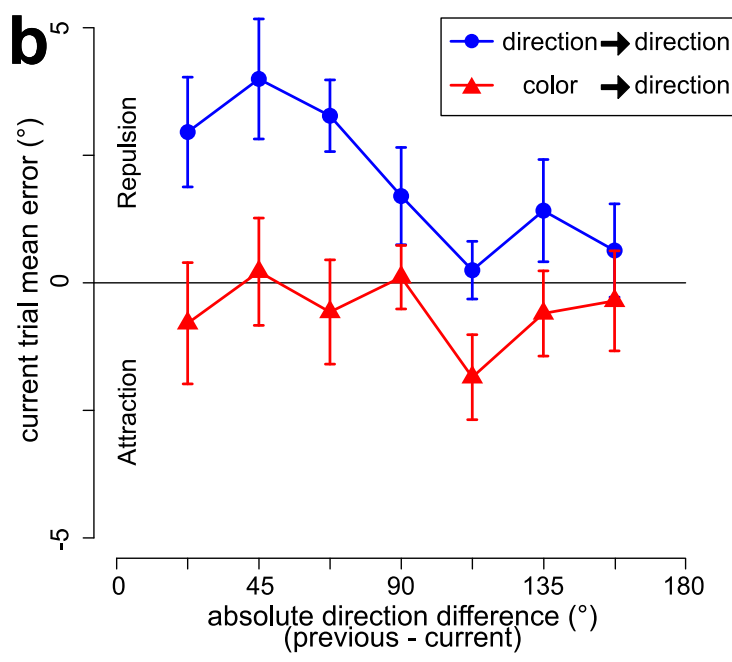

Fig. 2 Experiment 2 task and results. (a) Sequence of stimuli. The dots were always either pink or green, and the test options were also pink and green. (b) Mean response errors for the direction report as a function of the absolute direction difference between the current and previous trials, plotted separately for trials preceded by a direction report and trials

experimental trials ( 16 directions $\times 28$ trials). This included 224 direction-report trials intermixed with 224 color-report trials. Trials with a response error larger than $60^{\circ}(2.41 \%$ of the direction-report trials) were excluded.

\section{Results and discussion}

The mean accuracy for the color task was $96.9 \%(S E M=0.3)$, which was significantly greater than the mean accuracy for the color task in Experiment 1 (87.7\%) $[t(46)=-10.84, p=$ $2.934 \mathrm{e}-14]$.

As is shown in Fig. 2b, the reported direction on a given trial was biased away from the direction on the previous trial (for direction differences $<90^{\circ}$ ), but only when the preceding trial was a direction-report trial. Figure $2 \mathrm{c}$ shows the response errors averaged across trials with direction differences of less than $90^{\circ}$. One-sample $t$ tests against zero indicated that trials preceded by a direction report exhibited a significant repulsion bias $[t(23)=4.417, p=.0001$, Cohen's $d=1.275]$, whereas
Direction Report cue: $500 \mathrm{~ms}$

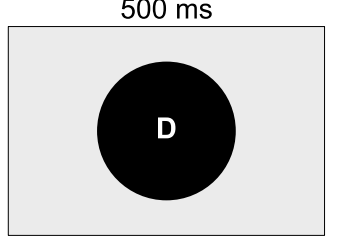

Color Report cue: $500 \mathrm{~ms}$

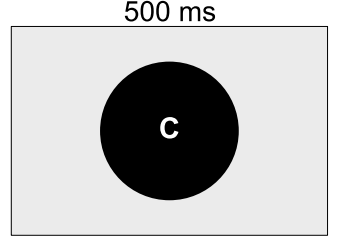

Button press (left or right)

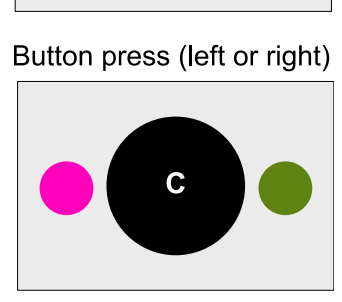

Direction estimation using a mouse
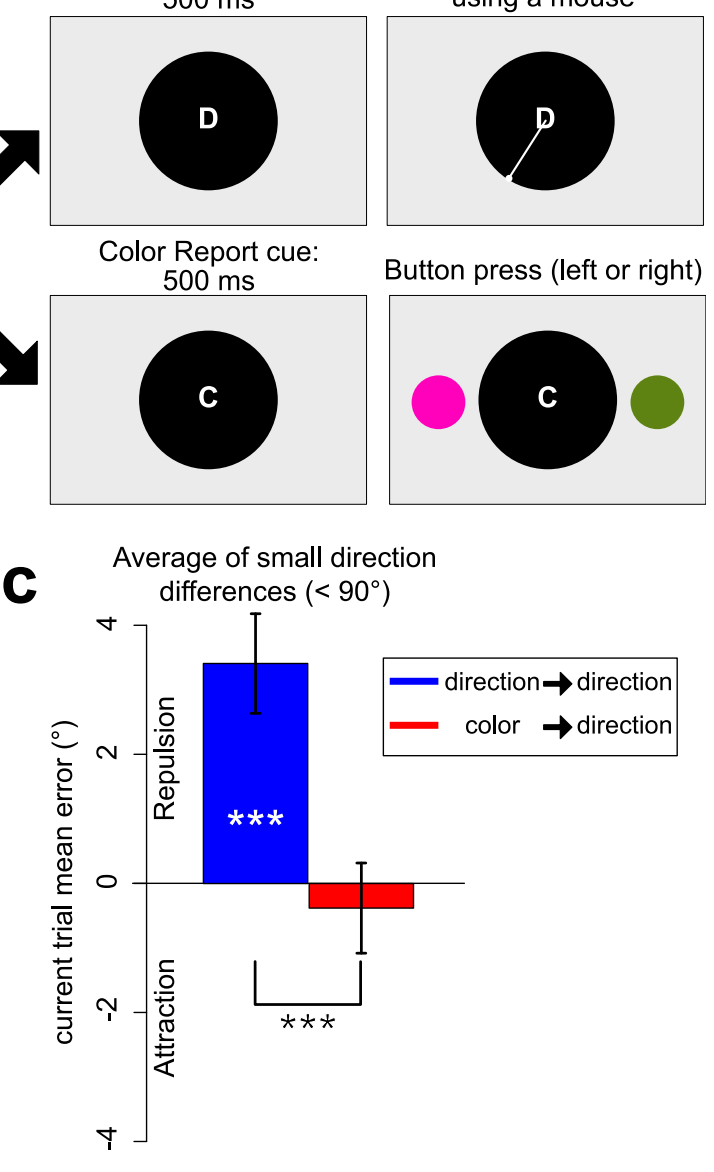

preceded by a color report. (c) Mean response errors for the direction report, averaged across small direction differences $\left(22.5^{\circ}-67.5^{\circ}\right)$, plotted separately for trials preceded by a direction report and trials preceded by a color report. Error bars indicate $\pm 1 S E M .{ }^{* * *} p<.001$.

trials preceded by a color report exhibited a small and nonsignificant attraction bias $[t(23)=-0.545, p=.590$, Cohen's $d=$ $-0.157]$. The serial dependence effect was significantly greater for trials preceded by a direction report than for trials preceded by a color report $[t(23)=3.691, p=.001$, Cohen's $d=$ 0.753].

These findings demonstrate that the main finding of Experiment $1-\mathrm{a}$ serial dependence for direction of motion only when the direction of motion was actually reported on the previous trial—is replicable and does not require a difficult color task.

\section{Experiment 3}

In Experiments 1 and 2, direction of motion was reported using a continuous estimation procedure, whereas color was reported with a $2 \mathrm{AFC}$ procedure. Thus, color-report and direction-report trials differed in the mode of the report as well 
as the content of the report. This raised the possibility that the lack of serial dependence in motion direction when the preceding trial was a color-response trial had been a result of the mode of the response on the preceding trial. Experiment 3 tested this possibility by using a delayed estimation procedure for both the color and motion reports (see Fig. 3a).

\section{Method}

The methods of Experiment 3 were identical to those of Experiment 2, except as noted. A new group of 24 college students participated (14 female, 10 male).

To parallel the use of 16 directions of motion, the colors of the dots in Experiment 3 were selected from a set of 16 colors (in steps of $\sim 22^{\circ}$ on the color wheel), drawn from the same color space used in Experiment 1. On color-report trials, a color wheel composed of 180 colors from this color space was presented around the black disk (Fig. 3a). Participants reported the perceived color of the dots from that trial by clicking the corresponding color on the color wheel with a mouse. The color wheel was randomly rotated every trial.

\section{Results and discussion}

The mean absolute error for the color report was $15.88^{\circ}$ (SEM $=0.64)$. Because the present study was designed to examine serial dependence in the motion report, our main analysis focused only on motion-report trials. However, as will be discussed later, our exploratory analysis on color-report trials revealed no clear evidence of serial dependence in the color report.

Figure $3 \mathrm{~b}$ shows the mean response errors for the motionreport trials. When motion was reported on the previous trial, the same repulsion pattern found in Experiments 1 and 2 was again observed, but this serial dependence was reduced when color was reported on the preceding trial. Figure $3 \mathrm{c}$ shows the
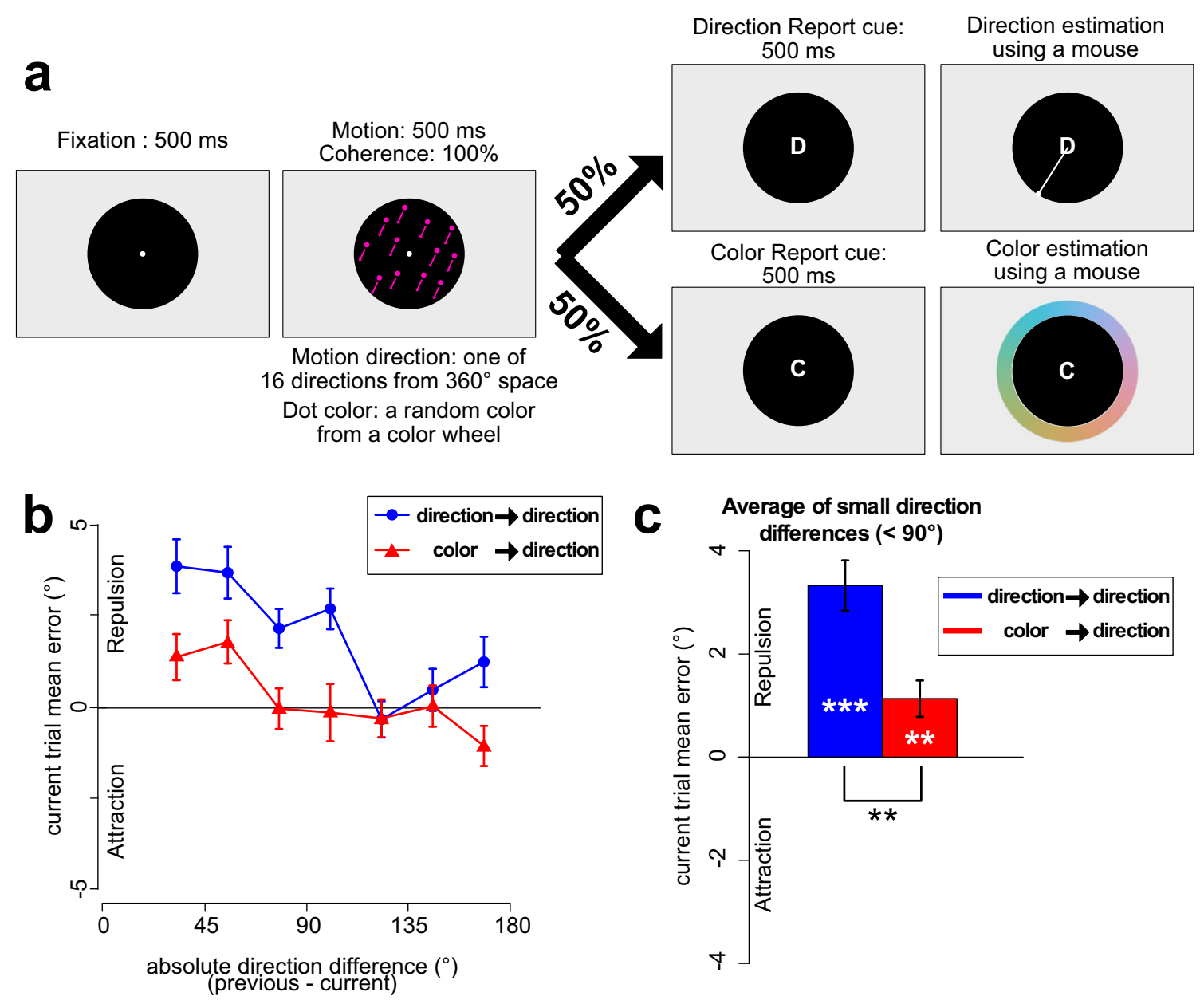

Fig. 3 Experiment 3 task and results. (a) Sequence of stimuli. The stimuli and task were identical to those of Experiment 1, except that participants reported the color (on color-report trials) by clicking on the color of the dots using a color wheel. (b) Mean response errors for the direction report as a function of the absolute direction difference between the current and previous trials, plotted separately for trials preceded by direction report

and trials preceded by color report. (c) Mean response errors for the direction report, averaged across small direction differences $\left(22.5^{\circ}-\right.$ $\left.67.5^{\circ}\right)$, plotted separately for trials preceded by a direction report and trials preceded by a color report. Error bars indicate \pm 1 SEM. ${ }^{* * *} p<.01$, ${ }^{* * * *} p<.001$. 
response errors averaged across trials with direction differences of less than $90^{\circ}$. One-sample $t$ tests against zero indicated that a significant repulsion bias was present in both the trials preceded by a direction report $[t(23)=7.081, p=$ $3.252 \mathrm{e}-07$, Cohen's $d=2.044$ ] and the trials preceded by a color report $[t(23)=3.204, p=.003$, Cohen's $d=0.924]$. However, a paired $t$ test indicated that the effect was weaker for trials preceded by a color report $\left(M=1.17^{\circ}\right)$ than for trials preceded by a direction report $\left(M=3.46^{\circ}\right)[t(23)=3.717, p=$ .001 , Cohen's $d=0.759$ ]. Thus, although a small serial dependence effect in the perception of motion direction could be observed when color rather than motion was reported on the previous trial, the serial dependence effect was more than twice as large when the direction rather than the color of the stimuli was reported on the preceding trial.

The presence of a small repulsion effect when color was reported on the previous trial in this experiment might reflect an automatic preparation of the motion direction response on a subset of the color-report trials. That is, because the response involved moving the mouse to a spatial location on both colorreport and motion-report trials, participants may have prepared the motion report even when cued to make a color report. Alternatively, it may have been driven by the more precise encoding of the color and/or the difficulty of the color task relative to the tasks used in Experiments 1 and 2. Future research will be necessary to investigate the source of the small serial dependence effect following color-report trials.

We also assessed whether the motion direction report on the current trial was influenced by the location of the mouse click when color was reported on the previous trial. We found a hint of an attraction toward the location of the previous-trial report (a mean bias of $-0.86^{\circ}$ when averaged across the $22.5^{\circ}-67.5^{\circ}$ direction differences), but this effect was not significantly different from zero $[t(23)=-1.866, p=.075]$.

\section{Discussion}

In the present study we investigated whether encoding the previous-trial stimulus is sufficient to produce serial dependence in motion perception or whether serial dependence depends on postencoding processes. We consistently found that serial dependence was reduced or eliminated when the direction information was not used on the previous trial, even though the task required the direction of motion to be perceived and stored in memory on every trial. Although it will be important to determine whether this pattern of results generalizes to other visual features, these results indicate that encoding a stimulus is not sufficient to produce serial dependence on the next trial, and that serial dependence can be modulated by processes associated with the $u$ se of the relevant feature information.
We do not conclude that reporting the relevant information on the previous trial is always necessary to induce a serial dependence effect. It may not be the report per se that produces serial dependence, but some other processes that accompany the report (e.g., decision processes and/or response preparation). If the manipulation of the previous-trial report does not completely eliminate this process, then some serial dependence will still be observed. Thus, the finding that the serial dependence effect was not completely eliminated in Experiment 3 following colorreport trials does not substantially weaken our main conclusion, which is that the mere encoding of the previous-trial stimulus is not sufficient for serial dependence to occur.

Additional research will be necessary to determine the specific mechanisms by which postencoding processes in the previous trial modulate serial dependence, but there are at least two general classes of possibilities. One is that making the response on one trial enhances or transforms the memory representation of the reported feature so that it has a stronger impact on the encoding or response on the next trial. Another possibility is that responding to one feature of the stimulus (e.g., color) disrupts the memory representation of the other dimension (e.g., direction of motion), so that it can no longer influence processing on the next trial (Anderson, Bjork, \& Bjork, 1994).

A recent study showed that serial dependence can also be observed for color (Barbosa \& Compte, 2018). Although the present study was not designed to investigate serial dependence in color perception, we explored whether color reports exhibited serial dependence in Experiment 3. We did not find clear evidence for a color serial dependence effect, irrespective of whether color or motion was reported on the previous trial. However, given the results reported by Barbosa and Compte, we are not concluding that serial dependence is generally absent for color: The specific task used in the present study might not have been optimal for inducing color serial dependence. Future research will be necessary to investigate this possibility.

Acknowledgment This research was made possible by grant R01MH076226 to S.J.L.

Open Practices Statement The data for all experiments are available at https://osf.io/g35jp. None of the experiments was preregistered.

Author Contributions G.B. designed the study with input from S.J.L. G.Y.B. oversaw data collection and analyzed the data. G.-Y.B. and S.J.L. wrote the manuscript.

\section{References}

Anderson, M. C., Bjork, R. A., \& Bjork, E. L. (1994). Remembering can cause forgetting: Retrieval dynamics in long-term memory. Journal of Experimental Psychology: Learning, Memory, and Cognition, 20, 1063-1087. https://doi.org/10.1037/0278-7393.20.5.1063 
Bae, G.-Y., \& Luck, S. J. (2017). Interactions between visual working memory representations. Attention, Perception, \& Psychophysics, 79, 2376-2395. https://doi.org/10.3758/s13414-017-1404-8

Bae, G.-Y., \& Luck, S. J. (2019). Reactivation of previous experiences in a working memory task. Psychological Science, 30, 587-595. https://doi.org/10.1177/0956797619830398

Barbosa, J., \& Compte, A. (2018). Build-up of serial dependence in color working memory: Supplementary figures. BioRxiv preprint. https:// doi.org/10.1101/503185

Brainard, D. H. (1997). The Psychophysics Toolbox. Spatial Vision, 10, 433-436. https://doi.org/10.1163/156856897X00357

Czoschke, S., Fischer, C., Beitner, J., Kaiser, J., \& Bledowski, C. (2019). Two types of serial dependence in visual working memory. British Journal of Psychology, 110, 256-267. https://doi.org/10.1111/bjop. 12349

Fischer, J., \& Whitney, D. (2014). Serial dependence in visual perception. Nature Neuroscience, 17, 738-743. https://doi.org/10.1038/nn.3689

Liberman, A., Fischer, J., \& Whitney, D. (2014). Serial dependence in the perception of faces. Current Biology, 24, 2569-2574. https://doi.org/ 10.1016/j.cub.2014.09.025
Papadimitriou, C., Ferdoash, A., \& Snyder, L. H. (2015). Ghosts in the machine: Memory interference from the previous trial. Journal of Neurophysiology, 113, 567-577. https://doi.org/10.1152/jn.00402. 2014

Pelli, D. G. (1997). The VideoToolbox software for visual psychophysics: Transforming numbers into movies. Spatial Vision, 10, 437-442. https://doi.org/10.1163/156856897X00366

Roitman, J. D., \& Shadlen, M. N. (2002). Response of neurons in the lateral intraparietal area during a combined visual discrimination reaction time task. Journal of Neuroscience, 22, 9475-9489. https://doi.org/10.1523/JNEUROSCI.22-21-09475.2002

Suárez-Pinilla, M., Seth, A. K., \& Roseboom, W. (2018). Serial dependence in the perception of visual variance. Journal of Vision, 18(7), 4. https://doi.org/10.1167/18.7.4

Publisher's note Springer Nature remains neutral with regard to jurisdictional claims in published maps and institutional affiliations. 